\title{
Los ingresos petroleros y el crecimiento económico en Ecuador (2000-2015)
}

\section{Oil revenues and economic growth in Ecuador (2000-2015)}

\author{
Pedro Cepeda Ch."', Eduardo Zurita M.!' y Dante Ayaviri N!") \\ (1) Facultad de Ciencias Políticas y Administrativas, Universidad Nacional de Chimborazo. Ecuador \\ Email: vdayaviri@gmail.com
}

\begin{abstract}
Resumen
El presente trabajo de investigación intenta explicar el crecimiento económico de Ecuador a partir de los ingresos del petróleo, dado que en los últimos diez años el sector petrolero y el crecimiento económico han llamado la atención en la literatura económica; su importancia se remite a la teoría de crecimiento de Solow. Las principales variables analizadas fueron los ingresos petroleros, como una variable determinante de la función de producción, la misma se estimó en términos per cápita por trabajador; para ello, se utilizaron series estadísticas del Banco Central de Ecuador y los mínimos cuadrados ordinarios; asimismo, la formación bruta de capital fijo, población económicamente activa y los ingresos petroleros, este último como una variable adicional ajena a la función de producción para explicar el crecimiento económico. Como principal resultado se obtiene una relación positiva del PIB por trabajador (y) con la formación bruta de capital fijo por trabajador $(k)$ y el ingreso petrolero por trabajador (yp), es decir cuando las variables inversión y renta aumentan, el PIB por trabajador también se incrementa.
\end{abstract}

Palabras claves: ingreso percápita, crecimiento económico, petróleo, inversión,

\begin{abstract}
This research attempts to explain economic growth in Ecuador from oil revenues, since in the last ten years the oil sector and economic growth have attracted attention in the literature; its importance refers to the theory of Solow growth. The main variables analyzed were oil revenues, as a key variable in the production function, it was estimated per capita terms per worker; for this, statistical series Central Bank of Ecuador and ordinary least squares were used; Also, the gross fixed capital formation, economically active population and oil revenues, the latter as a foreign additional variable production function to explain economic growth. As a main result a positive relationship GDP per worker ( $y$ ) with the gross fixed capital formation per worker $(k)$ and oil income per worker (yp) is obtained, when the variables investment and income increase, GDP per worker also increases.
\end{abstract}

Keywords: per capita income, economic growth, petroleum, investment. 


\section{Introducción}

El crecimiento económico ha sido uno de los temas más estudiados y discutidos en la academia y los centros de investigación, las variables más analizadas son la acumulación de capital físico, mejora del capital humano, progresos tecnológicos, comercio exterior y recursos naturales. Los debates e interpretaciones han venido de diferentes escuelas del pensamiento económico, buscando dar respuestas a los fenómenos socioeconómicos y a las inquietudes intelectuales (Romer, P. 1993; Tödtling F, y Trippl, M. 2005; Gadrey J, y Jany-Catrice, F. 2006; Stiglitz, J. 2012). Existe la corriente de la "maldición de recursos naturales", al respecto Karl (1997) señala que los países con mayores riquezas como del petróleo, en el largo plazo no tienen un crecimiento sostenido esto porque no priorizan otros sectores como la innovación y el conocimiento. Existen una serie de justificaciones que tratan de explicar esto, así para Sachs J. D. y Warner, A M. (1997) la abundancia de recursos tienden a generar corrupción e ineficiencias en las burocracias, además de que los gobiernos que controlan ingresos provenientes de recursos naturales tienden a desperdiciar en consumos innecesarios. Para Karl, T. (1997) las economías dependientes de exportaciones de mercancías primarias influyen en las formas de sus instituciones sociales como políticas. Por otra parte, existe la teoría de la enfermedad holandesa que se experimenta en Holanda tras el descubrimiento de petróleo en el Mar del Norte en la década de los sesenta, que explica los efectos negativos que puede traer la mayor dependencia de los recursos naturales, como es la apreciación de la tasa de cambio real, generando así la contracción más allá de lo normal de los sectores de bienes comerciables, sean exportables o importables (Olusi, J.O y Olagunju, M. A. 2005); definitivamente, el desarrollo de un país podría radicar en el uso eficiente o no, de los recursos naturales y de forma sostenida, el mismo repercute en la economía y el bienestar de la población (Krugman, P. 1998; Mehlum et al, 2006; Murshed, S. M. 2004; Olusi J. O. y Olagunju, M. A. 2005; Ross, M. L. 1999; Sachs, J. D. y Warner, A. M. 1997) y en consecuencia repercute de forma directa en el crecimiento económico en primera instancia y posteriormente un impacto social (Krugman, P. 2000; Domar, E. 2004; Barro, R. J. y Sala-i-Martin, X. 2004; Barbera, R. A. y Doncel, L. M. 2009; Acemoglu, D. 2009).

En este contexto, la producción del petróleo para Ecuador es de extrema importancia en el ámbito del desarrollo económico y social, y en consecuencia el presente estudio pretende acercarse al fenómeno de los ingresos económicos y su efecto en el crecimiento económico; por tanto, intenta comprender el comportamiento del crecimiento económico del Ecuador, ante los cambios cíclicos de los ingresos por concepto del petróleo en el periodo de estudio, influenciado principalmente de los shocks internacionales, y los cambios en los precios de las materias primas principalmente. Estos shocks han obligado al Estado que replantee diversas políticas para afrontar los desequilibrios como son: el cambio de la matriz productiva y educativa; y la ley de Hidrocarburos, entre otras.

\section{Materiales y métodos}

Según López, et al. (2013) menciona que la renta es cualquier pago al propietario del recurso natural que queda después de remunerar al trabajo (incluyendo al calificado), al capital (incluyendo la tecnología). Desde este planteamiento el ingreso petrolero es la renta por el derecho de uso del recurso, las cuales dependerá de los factores endógenos y exógenos, es decir del comportamiento de la economía mundial y de las políticas nacionales. El crecimiento económico es función del capital, trabajo, recursos naturales, y tecnología como las fuentes principales para el aumento del PIB (Lucas, R. 1988; Samuelson, P. y Nordhaus, W. 20 I0), así los recursos naturales son una de las fuentes principales para los paises en desarrollo. Pero estas rentas no les han garantizado un crecimiento economico sostenido, mas bien, ha sido de un gran perjuicio economico, porque hace que sean mas dependientes en las finanzas publicas y en las exportaciones (Stiglitz, P. 20 I2; Snowdon, B. y Vane, H. R. 2005). En este contexto, a continuación se describe sucintamente algunas cuestiones teóricas vinculadas a los modelos y cuestiones técnicas aplicadas.

\section{Modelo de crecimiento de Solow}

El modelo de crecimiento económico de Solow R. (1956), se basa en una función de producción neoclásica, donde el producto depende de la combinación de trabajo y capital y utiliza los típicos supuestos neoclásicos, productividad marginal decreciente, competencia perfecta y su principal conclusión es que las economías alcanzarán un estado estacionario en el cual el crecimiento del producto per cápita es nulo (Lomelí, $H$. y Rumbos, B. 2003). Así, se aplica el modelo que explica la existencia de tres fuentes principales, entre las cuales están: la población, capital y la tecnología.

$$
Y=A f(K, L)
$$

Esta función de producción se puede transformar en términos per cápita, para lo cual se parte del supuesto de que el crecimiento de la población es constante.

$$
y=a f(k) ; \text { Dónde: } y=Y / L ; k=K / L
$$

Y, partiendo del supuesto de una economía cerrada y en ausencia del cambio tecnológico, se tiene que la inversión es igual ahorro ( $\mid=S)$, de esta forma, desa- 
parece el problema de un posible exceso o insuficiencia de la demanda que juega un papel fundamental en la teoría keynesiana, por otro lado, la depreciación es una proporción fija del capital (k). Igualmente supone que el ahorro es una proporción del producto sy. De esta forma, un cambio en el stock de capital viene dado por:

$$
\Delta k=s y-\delta k
$$

En consecuencia, es el incremento del capital per cápita; producción per cápita; y tasa de depreciación. También el modelo predice un equilibrio único, definido como un estado estacionario en el cual $k=y$ $=0$. En el estado estacionario la tasa de ahorro permite financiar una inversión tal que contrarresta exactamente la caída en el stock de capital per cápita asociado a la depreciación y al crecimiento poblacional, en ausencia de progreso tecnológico. En el modelo básico la producción depende de la combinación de varios factores productivos. Sin embargo, la inclusión de la variable ingresos petroleros en el análisis da lugar a la consideración del fenómeno de la renta analizado previamente, con consecuencias importantes sobre la formulación del modelo. Ahora bien, ¿qué papel juega la inclusión del ingreso petrolero en el modelo? Para lo cual, se analiza los trabajos realizados de Schlisser, J. y Silva, C. (2000) y Hernández, E. (2006). En la cuales parte de la función de producción presentada anteriormente (2), y se incluye la renta como variable adicional, a su vez no depende de ninguna otra variable, más bien se relaciona como un ingreso de recurso natural que es una de las fuentes principales del crecimiento económico. Esta variable está conectada directamente con el crecimiento económico, ya que si se incrementa el ingreso petrolero, aumentara también la inversión y el gasto público y como efecto también el crecimiento económico. Quedando en términos per cápita así:

$$
y=a f(k)+y p
$$

En donde, como se mencionó anteriormente la tecnología es constante, mientras que la producción (y), inversión (k) e ingreso petrolero (yp) varían. Dado que el ingreso del petróleo ecuatoriano ha sido destinado para dinamizar la economía por medio del gasto del Estado, la función de acumulación presentada anteriormente (3) se sufrirá una modificación al incorporar dicha renta, la cual se expresa en términos per cápita:

$$
\Delta k=\beta y p+s y-\delta k
$$

La inclusión de la renta petrolera tiene una significación importante, dado que el supuesto estado estacionario donde $k=y=0$, un incremento del ingreso petrolero con un producirá un incremento en la acumulación de capital, y por ende mayor crecimiento económico (Hernández, 2006).

\section{Aspectos Metodológicos.}

Para estimar la función de producción de Solow para el caso ecuatoriano, se utilizaron series estadísticas del Banco Central, y a través de la metodología de mínimos cuadrados ordinarios, se analizó las propiedades estadísticas de cada variable. A partir de la teoría económica del modelo, se incorpora las variables: formación bruta de capital fijo (FBKF), población económicamente activa (PEA) y el ingreso petrolero (yp), este último como una variable adicional ajena a la función de producción para explicar el crecimiento económico. Para el cálculo del modelo se utilizó el software econométrico Eviews. Se considera como periodo de análisis 2000-20 I 5, los datos son trimestrales con un total de 60 observaciones, también se trimestralizó la población económicamente activa debido a la inexistencia de fuentes estadísticas oficiales.

Retomando la teoría económica descrita anteriormente de manera formal se conoce que el PIB tiene una relación directa con la formación bruta de capital fijo y el ingreso petrolero. Al utilizar el modelo de crecimiento de Solow se desarrolla una ecuación logarítmica de elasticidades constantes, y para el desarrollo de la misma se emplea supuestos que determinan los parámetros a explicar: una economía cerrada, el ahorro es exactamente igual a la inversión, la población crece a la misma tasa de fuerza de trabajo (PEA) y tecnología constante.

Así, a partir de la ecuación (4), que el PIB por trabajador está en función de la inversión por trabajador e ingreso petrolero, y dejando como constante la tecnología. Para el presente caso, para calcular en términos per cápita por trabajador, se divide entre población económicamente activa (PEA). Siguiendo estos lineamientos la ecuación es la siguiente:

$$
\Delta \ln \left(\frac{Y}{L}\right)=\beta_{0}+\beta_{1} \Delta \ln \left(\frac{K}{L}\right)+\beta_{2} \Delta \ln \left(\frac{Y_{p}}{L}\right)+e
$$

En términos per cápita se expresa así:

$$
\Delta \ln (y)=\beta o_{t}+\beta 1 \Delta \ln \left(k_{\mathrm{t}}\right)+\beta 2 \Delta \ln \left(y p_{\mathrm{t}}\right)+e_{\mathrm{t}}
$$

Donde:

y= Producto Interno Bruto per cápita.

kt= Formación Bruta de Capital Fijo por trabajador.

$\mathrm{ypt}=$ Ingreso petrolero por trabajador. 
et $=$ Error.

$\beta 0, \beta 1, \beta 2$ : Parámetros de regresión a estimar por el método de mínimos cuadrados.

\section{Resultados}

\section{Ingreso petrolero por trabajador}

El ingreso petrolero por trabajador en el periodo de estudio creció a una tasa promedio de 7,76\%.

Uno de los años con mayor crecimiento fue 2004, cre ciendo en valor monetario por trabajador a $\$ 100,06$, re presentando un aumento de $6,64 \%$ con respecto al año anterior, esto debido a la puesta en marcha del nuevo Oleoducto de Crudos Pesados (OPC), que fue construido por un consorcio privado liderado por Techint (Argentina) y entró en funcionamiento en 2003, donde Ecuador participa ampliando su capacidad de transportación y generando mayor producción y exportación de petróleo.

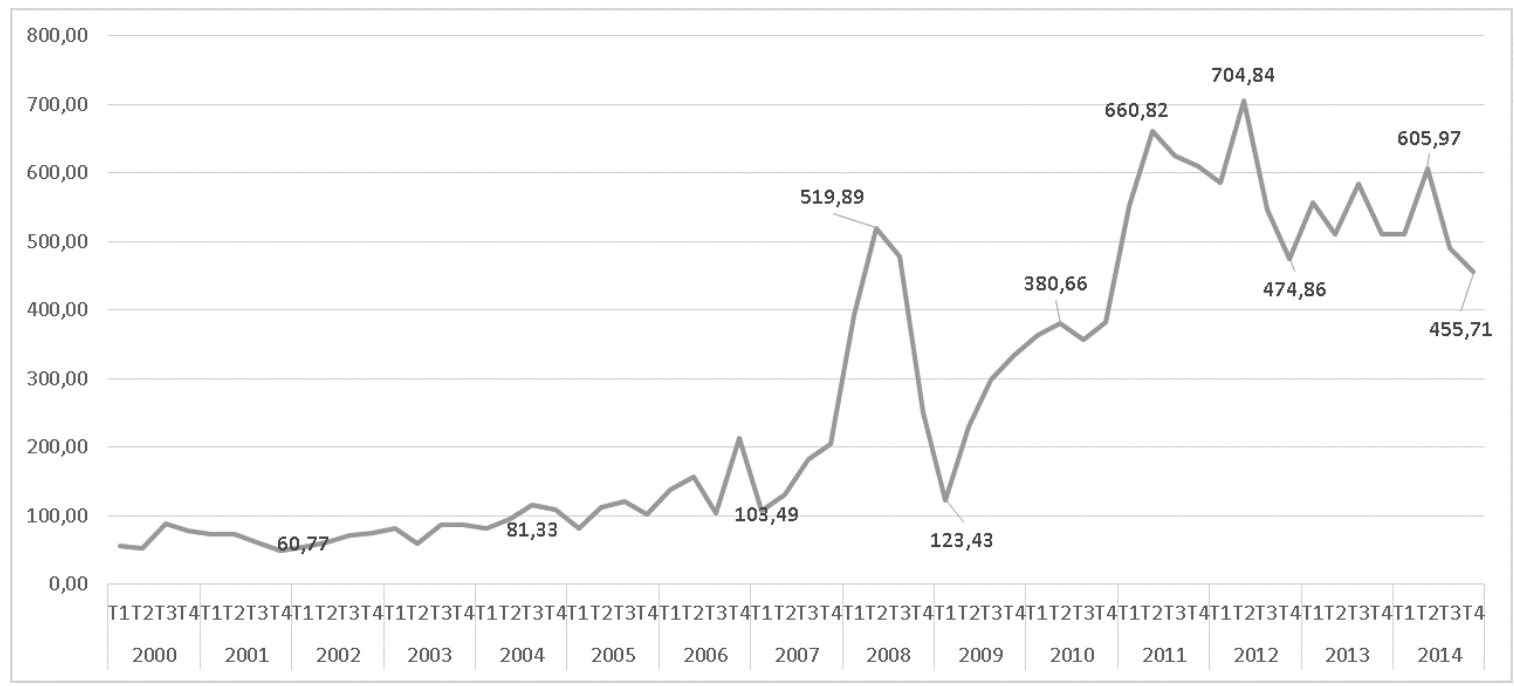

Figura I. Ingreso por trabajador periodo trimestral 2000 - 20I4. Expresado en dólares

Fuente: Elaboración propia con base a datos de Banco Central del Ecuador, 2015

En el segundo trimestre del 2008 el ingreso petrolero por trabajador creció a niveles inesperados alcanzando a \$519,89 por trabajador, con una tasa de crecimiento de $32,56 \%$ en comparación con el trimestre anterior, esto debido al incremento del precio del petróleo a nivel internacional que alcanzó los \$100,06, registrando así uno de los precios records en el periodo de estudio.

Los años 2011 y 2012 también experimentó un crecimiento sostenido, registrando para el segundo trimestre del 2012, $\$ 704,84$, obteniendo un crecimiento en relación al trimestre anterior en un $20,42 \%$, esto principalmente al buen desempeño de la economía mundial, donde el precio de los comodities se mantuvo por encima de los 90 dólares americanos. Pero, cabe recalcar, en estos años el Estado controlo el 71\% de la producción petrolera, esto debido a la normativa legal de la ley hidrocarburos donde los contratos pasan a ser únicamente de prestación de servicios, en la cual gobierno paga una cantidad fija.

Al contrario, el peor desempeño económico fue el último trimestre del 2008 y el primer trimestre del 2009 , donde esta variable disminuyó en un $-47,36 \%$ y $-50,92 \%$ respectivamente, este decrecimiento se produjo debido al crisis financiera internacional, donde afecto el precio del petróleo West Texas Intermediate (WTI), disminuyendo a $\$ 61,92$, es decir disminuyendo en un $38 \%$ con respecto al año anterior. Así también, en el tercero y cuarto trimestre del último año de estudio existe un decrecimiento, esto debido al desaceleramiento de las economías emergentes, especialmente China y también a la apreciación del dólar, donde como se ve en el gráfico $N^{\circ}$ I, el ingreso petrolero por trabajador decrece a $\$ 455,71$, es decir, en valores porcentuales - 19,04\% y -7, I I por ciento respectivamente.

Para el último período de estudio el desempeño fue inferior, esto debido a las malas condiciones de la economía internacional, donde incidieron los precios de las materias primas, principal ingreso para la economía ecuatoriana, por su dependencia de las exportaciones de bienes primarios, que actualmente representa el $56 \%$ con un tasa de crecimiento promedio anual de 14\% en el periodo de estudio.

\section{PIB por trabajador}

El promedio trimestral en el periodo de análisis fue de 3,30\%, registra una tendencia; de igual forma, se observa que en el primer trimestre del 2008 tuvo un crecimiento importante en comparación a todos los trimestres analizados, alcanzando a 8,85\%, aunque menor a lo experimentado en los trimestres del año 2000. 


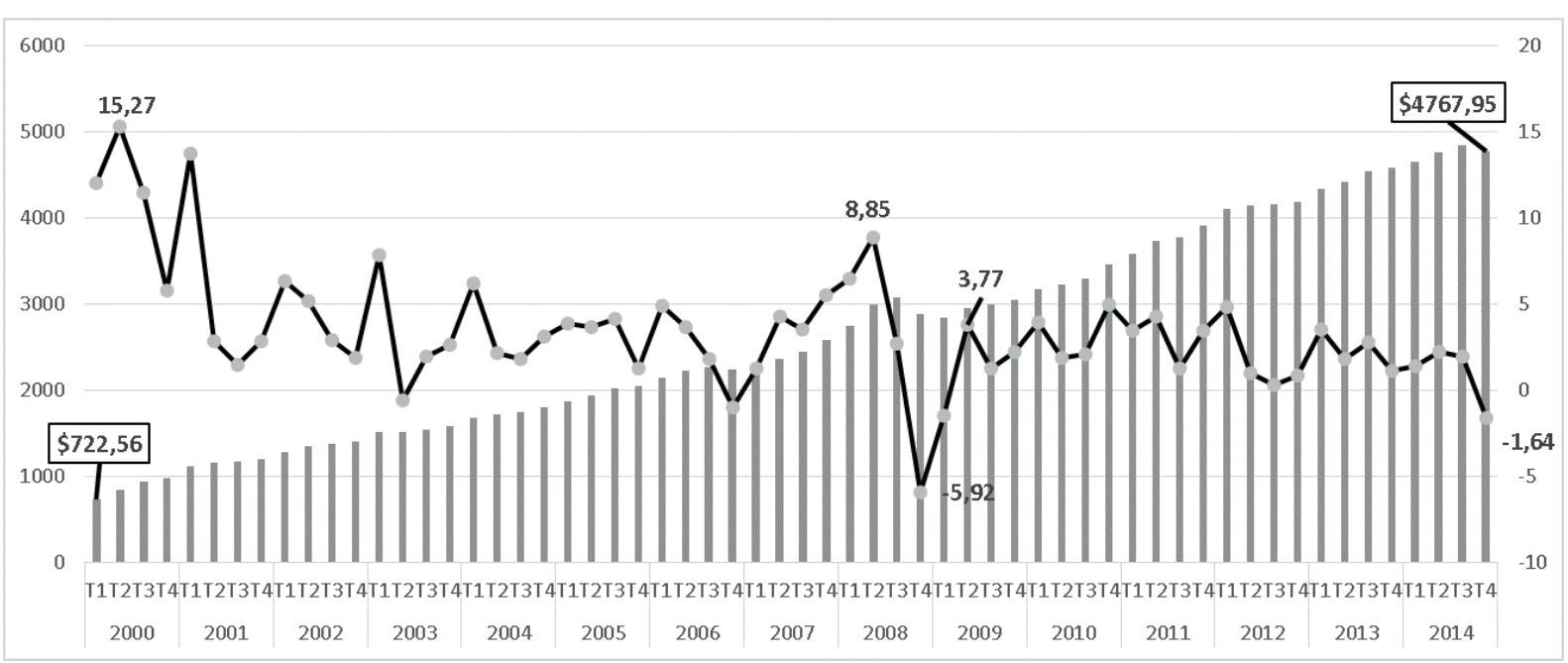

Figura 2. PIB por trabajador periodo trimestral 2000 - 20I4. Expresado en dólares

Fuente: Elaboración propia con base a datos de Banco Central del Ecuador, 2015

Como se aprecia en el gráfico, los últimos trimestres en el periodo 2008 y 2014, registran tasas de crecimiento negativas, el primero debido a la crisis financiera internacional y la segunda debido al debilitamiento de las economías emergentes que afectó el consumo de las materias primas, fuente primarias que generan divisas al Ecuador y por la apreciación del dólar. En lo que respecta a valores monetarios, en el mismo periodo, el PIB por trabajador creció de $\$ 69,06$ a $\$ 515,80$, es decir creciendo en un 647\% (BCE, 20I5).

Este desempeño se debe al crecimiento ascendente que ha sufrido el PIB real después de la dolarización, esto principalmente por el buen desempeño de la economía internacional y la creciente demanda de las materias primas, especialmente el petróleo.

\section{FBKF por trabajador}

Según el BCE (20 I5), la Formación Bruta de Capital Fijo (FBKF) comprende a la inversión de un país, y representada por la variación de los activos fijos no financieros tanto privados como públicos, (total de adquisiciones menos ventas de activos fijos), en un período de tiempo determinado. Y es considerada como el motor del crecimiento económico debido a que permite incrementar la capacidad productiva de un país por varios períodos. Así, la FBKF por trabajador presenta un incremento del 3,98\% en el periodo de estudio, el mismo se debe al buen desempeño del sector petrolero y no petrolero, esto por el incremento de precios y la demanda de los comodities, que generó mayor ingreso para el Estado y en consecuencia, incrementó la inversión bruta.

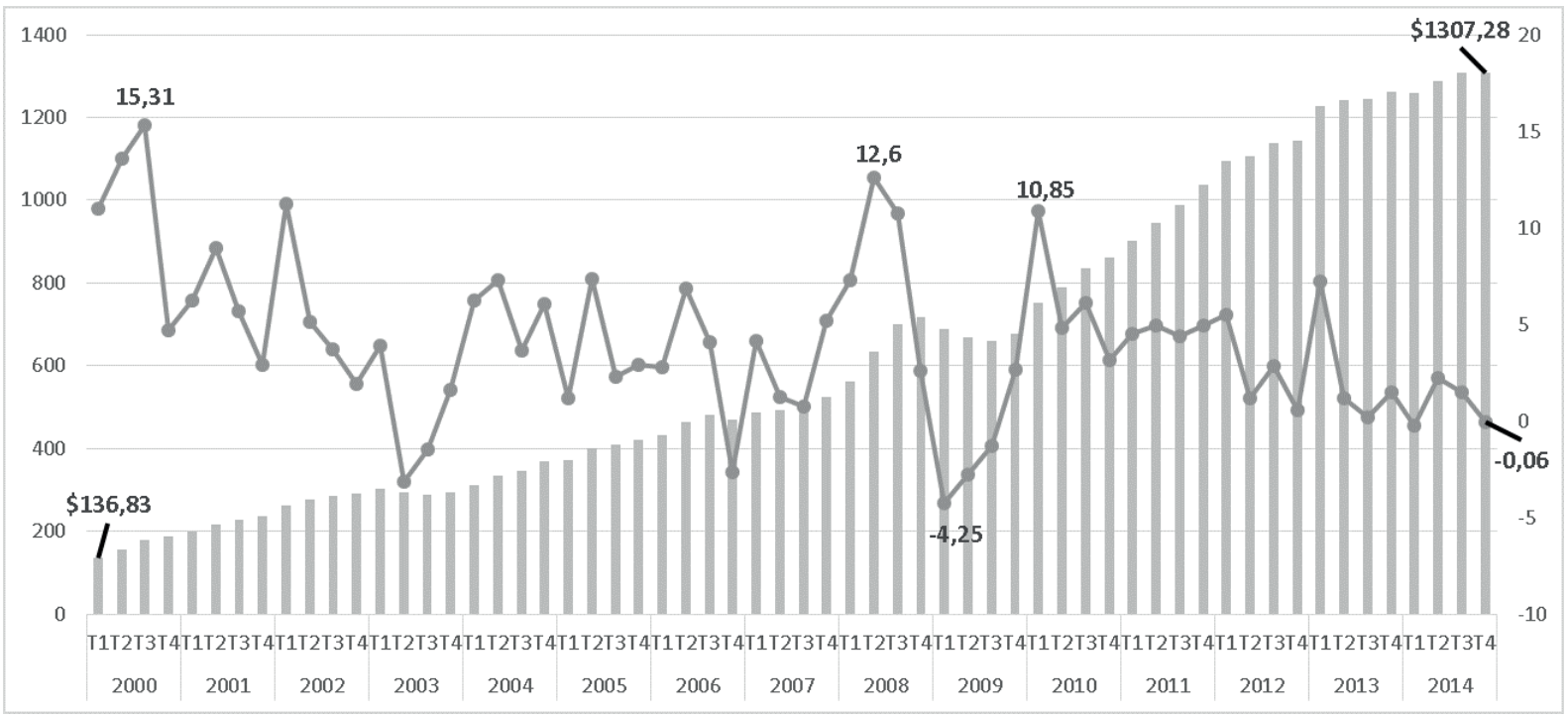

Figura 3. FBKF por trabajador periodo trimestral 2000 - 20I4. Expresado en porcentaje/dólares

Fuente: Elaboración propia con base a datos de Banco Central del Ecuador, 2015 
En el periodo de estudio, desde el primer trimestre del 2000 hasta el último trimestre del 2014 la inversión bruta creció en valores monetarios de \$136,83 a $\$ 1307,28$ por trabajador, es decir creció en un $855,4 \%$. Los trimestres con peor desempeño fueron como se puede apreciar en el grafico $\mathrm{N}^{\circ} 3$ el primer trimestre del 2009 , cayendo en $-4,25 \%$, y el último trimestre del 2014 disminuyendo en $-0,06 \%$, esto después de experimentar altas tasas de crecimiento, tanto en el 2008 y en el período 20 I I-20 I 3. Analizando las tres variables en conjunto, se puede apreciar en los gráficos que las variaciones son similares, es decir si la variable ingreso petrolero disminuye, las dos variables también decrecen.

En su promedio trimestral la FBKF por trabajador aporto con 22,50\% al PIB per cápita, mientras que el ingreso petrolero aportó con el 9\%. Al comparar las tasas de crecimiento del período, el mejor desempeño tuvo el sector petrolero con 7,7\%, mientras que la inversión bruta alcanzó un 4, $1 \%$ y el PIB per-cápita creció en promedio de $3,3 \%$.

\section{Aplicación y resultados de modelo econométri- co}

Para la aplicación del modelo, se desarrolló diversas pruebas econométricas de acuerdo a los lineamientos del método de mínimos cuadrados ordinarios (MCO), como: linealidad, estacionalidad, heterocedasticidad, normalidad y de no auto correlación, con la finalidad de no presentar una regresión espuria. Y, por otra parte, se procedió a realizar la prueba de estacionalidad con el test de Dickey Fuller Aumentado (ADF) para analizar el estado de las variables, en la cual los resultados arrojados por el test están en la Tabla I. De acuerdo al sustento teórico, el valor del test de ADF se sitúa al lado izquierdo del valor crítico, esto en todas las variables comprobadas, por lo que se puede concluir que las series son estacionarias en primeras diferencias.

\section{Tabla I.Test de Dickey-Fuller aumentado}

\begin{tabular}{lcc} 
Variable & $\begin{array}{c}\text { valor en primera } \\
\text { diferencia }\end{array}$ & Valor crítico de 5\% \\
\hline $\ln (y)$ & $-5,75$ & $-2,9 \mid$ \\
\hline $\ln (k)$ & $-4,98$ & $-2,9 \mid$ \\
\hline $\ln \left(y_{p}\right)$ & $-8,75$ & $-2,9 \mid$ \\
\hline
\end{tabular}

Fuente: Elaboración propia

Posteriormente, se procedió a realizar la regresión en primeras diferencias como se muestra en la Tabla I, utilizando el método de $\mathrm{MCO}$, en la cual se analiza los diversos supuestos del método. A continuación se detalla los principales resultados:
Tabla 2. Regresión de la función de producción por trabajador

\begin{tabular}{lcc} 
Variables & En primeras diferencias \\
$\ln (\mathrm{y})$ & & $\begin{array}{c}0.01198 \\
(2.729505)^{*}\end{array}$ \\
\hline $\ln (\mathrm{k})$ & 0.4942 & $(6.040946)^{*}$ \\
\hline $\ln \left(\mathrm{y}_{\mathrm{p}}\right)$ & 0.0305 & $(2.750057)^{*}$ \\
\hline $\mathrm{R}^{2}$ & & $0.448028(45 \%)$ \\
\hline $\mathrm{DW}$ & & 1.724439 \\
\hline
\end{tabular}

Fuente: Elaboración propia

*Son los valores de t-Statistic

Los coeficientes son significativos al 5\%

La ecuación en primeras diferencias muestra que, si la inversión por trabajador incrementa en un 1\%, el PIB crecerá en 0,49 por ciento; y si aumenta el I\% del ingreso petrolero por trabajador, el PIB por trabajador crecerá en $0,03 \%$, esto con una capacidad explicativa de $45 \%$ de las variables analizadas. Los resultados obtenidos tienen una relación con la teoría económica, demostrando una relación positiva de la producción por trabajador; es decir, si aumenta la inversión y el ingreso petrolero (ambos por trabajador), entonces el PIB por trabajador también crecerá en mayor o menor proporción. En consecuencia, se demuestra que en el Ecuador, tanto los ingresos petroleros y la formación bruta de capital fijo por trabajador, sí coadyuvaron al crecimiento económico en el periodo de estudio.

\section{Discusión}

La distribución de la renta petrolera en el caso ecuatoriano se ha llevado a cabo utilizando instrumentos de política asistencial, productiva y de desarrollo de infraestructuras públicas (puentes, hospitales, carreteras, hidroeléctricos y etc.). Esta particular distribución de la renta privilegió sensiblemente al gasto de inversión y muy poco a la generación de tecnología y de valor agregado. El Estado ecuatoriano ha priorizado el crecimiento a través del mercado interno, donde el consumo y la inversión pública fueron las que movilizaron la económica, esto se ha podido lograr por el incremento de los ingresos públicos del Gobierno, impulsado por el incremento del precio de petróleo en el mercado internacional, el cambio a la ley de hidrocarburos y reformas tributarias, la cual generó una expansión en la industria petrolera, y otras industrias que ofertan los servicios.

En los últimos años, Ecuador se ha enfocado a dinamizar la economía a través del gasto público, en las cuales se menciona: las inversiones en carreteras, las 
hidroeléctricas, seguridad, salud, educación, entre otros. Esto ha ocasionado que en el tiempo se incremente la inversión bruta por persona y el PIB per cápita. A menara de discusión, si bien es importante conocer las políticas del gobierno, éste ha priorizado la política fiscal como un instrumento dinamizador de la economía, por lo que los ingresos extraordinarios del petróleo han sido destinados al incremento del gasto público, enfocado más a la inversión pública. Los resultados al que aborda la presente investigación, demuestran que la economía está basada definitivamente en este sector. ¿Si el petróleo tiene un buen desempeño a nivel internacional, se podría seguir usando estos recursos para dinamizar la economía y tener un crecimiento económico sostenido en el largo plazo?

\section{Conclusiones}

En el periodo comprendido entre el 2000 y 2014 , se ha observado una continua aceleración en el ritmo de crecimiento del PIB por trabajador en el Ecuador, coincidiendo con el mayor dinamismo mostrado tanto en acumulación de capital físico como en las exportaciones de bienes y servicios. Tal aceleración en el ritmo de crecimiento del PIB por trabajador se explica fundamentalmente, por el incremento progresivo de la renta petrolera, lo cual ha mejorado los distintos mecanismos impulsores de acumulación de capital físico, siendo ésta la influencia causal más importante del crecimiento económico experimentado en el país a lo largo del período de estudio.

Se puede concluir señalando, que a medida que aumenta el ingreso petrolero por trabajador, las otras variables de estudio antes mencionadas en el tiempo sufrirán un incremento, dependiendo de las condiciones económicas y los precios internacionales. Centrando en los resultados de la ecuación de la función de producción las variables de estudio han explicado en un 45\% el crecimiento económico por trabajador, demostrando también que estos indicadores son significativos al 5\%. Lo cual es muy significante y bueno para contrastar de que si existe una fuerte causalidad de estas dos variables con el PIB per-cápita, y de esa manera se evidencia el planteamiento inicial, de que los recursos naturales sí generan un crecimiento económico. Sin embargo es necesario indicar que existen otros factores productivos que explican también este incremento en el PIB per-cápita, entre los cuales está el capital humano y el nivel tecnológico.

\section{Referencias}

Acemoglu, D. (2009). Introduction to Modern Economic Growth, Princeton University Press.

Barbera, R. A. \& Doncel L. M. (2009). La moderna economía del crecimiento, Editorial
Síntesis.

Barro, R. J. \& X. Sala-i-Martin. (2004), Economic Growth, MIT Press.

Domar, E. (2004). "Capital Expansion, Rate of Growth and Employment", Econometrica 14, (2004), pp. |37-| 47 .

Gadrey, J. \& Jany-Catrice, F. (2006). The New Indicators of Well-Being and Development, Palgrave, Houndmills.

Hernández, E. (2006). Renta petrolera y su impacto en crecimiento económico de Venezuela, Revista latinoamericana de desarrollo, Vol. 37, N. 147

Karl, T. (1997). The Paradox of Plenty. Oil Booms and Petro States, Berkeley, University of California Press.

Krugman, P. (1998). The Role of Geography in Development. Annual World Bank Conference on Development Economics, Washington D. C.

Krugman, P. (2000). El retorno de la economía de la depresión, Editorial Crítica.

López, E., Montes, E., \& Garavito, A. (20 I 2). La economia petrolera en Colombia. Bogota: Borradores de economía. Banco Central de Colombia.

López, E., Montes, E., Garavito, A., \& Collazos. (20|3). La economia petrolera en Colombia, (Parte II). Bogotá.: Borradores de economia.Banco Central de Colombia.

Lomelí, H., \& Rumbos, B. (2003). Métodos dinámicos en economía, Thomson Editores.

Lucas, R. (1988). "On the Mechanics of Economic Development", Journal of Monetary Economics

22 ( 1 988), pp. 3-42.

Mehlum, H., Moene, K., \& Torvik, R. (2006). Institutions and the Resource Curse. The Economic Journal, II6 (January), I-20.

Murshed, S. M. (2004). When does natural resource abundance lead to a resource curse?. EEP Discussion Paper 04-0 I. International Institute for Environment and Development, London.

Olusi J. O. \& Olagunju M. A. (2005). The Primary Sectors of the Economy and the Dutch Disease in Nigeria. The Pakistan Development Review , 44:2, I59-175

Romer, P. (1993). Idea gaps and object gaps in economic development. Journal of Monetary Economics, 
$32,543-573$.

Ross, M. L. (1999). The Political Economy of The Resource Curse. World Politics, 5I, 297-322.

Sachs, J. D. \& Warner, A. M. (1997). Natural Resource Abundance and Economic Growth. Harvard University, Cambridge MA.

Schliesser, J. \& Silva, C. (2000), La renta petrolera y crecimiento económico de Venezuela, BCV.

Samuelson, P., \& Nordhaus, W. (20 I0). Macroeconomía con aplicaciones a Latinoamerica (19a ed.). Mexico D.F., Mexico: Mc Graw

Snowdon, B. \& Vane H. R. (2005). Modern Macroeconomics, Edward Elgar Publishing.

Stiglitz, J. (20/2). The Price of Inequality: How Today's Divided Society Endangers Our Future, W. W. Norton \& Company.

Solow, R. (1956). "A Contribution to the Theory of Economic Growth", Quarterly Journal of Economics, No 70, págs. 65-94.

Tödtling, F. \& Trippl, M. (2005). One size fits all? Towards a differentiated regional innovation policy approach, Research Policy, 34, 1023-1 209. 